\title{
Disturbance-Observer-Based Hysteresis Compensation for Piezoelectric Actuators
}

\author{
Jingang Yi, Senior Member, IEEE, Steven Chang, and Yantao Shen, Member, IEEE
}

\begin{abstract}
We present a novel hysteresis compensation method for piezoelectric actuators. We consider the hysteresis nonlinearity of the actuator as a disturbance over a linear system. A disturbance observer (DOB) is then utilized to estimate and compensate for the hysteresis nonlinearity. In contrast to the existing inverse-modelbased approach, the DOB-based hysteresis compensation does not rely on any particular hysteresis model, and therefore provides a simple and effective compensation mechanism. We design and fabricate a lead magnesium niobate-lead titanate (PMN-PT) piezoelectric actuator for microscale tip-based power sintering process. Experimental validation of the proposed hysteresis compensation is performed on the PMN-PT cantilever piezoelectric actuator. The experimental results demonstrate the effectiveness and efficiency of the approach.
\end{abstract}

Index Terms-Compensation, disturbance observer (DOB), hysteresis, piezoelectric actuator, smart materials.

\section{INTRODUCTION}

D URING the last decade, micro/nanomanipulation have been extensively studied in robotics and control communities [1], [2]. For manipulation platforms, for example, such as that used in atomic force microscopy (AFM), piezoelectric cantilever beams and piezoelectric tubes are the most widely used actuation mechanisms. However, one of the potential issues of using these actuators is poor performance and limited bandwidth due to the nonlinear hysteresis effect of the piezoelectric materials [3].

To compensate for hysteresis nonlinearity, various control strategies have been proposed in the past decade. A review of the hysteresis modeling and compensation methods for micro/nanoapplications can be found in recent two special issues in control engineering community [3], [4]. Two control structures are mainly used for hysteresis compensation [5], [6]: 1) an inverse-model feedforward hysteresis compensation and 2) a closed-loop feedback hysteresis compensation. The inversemodel feedforward hysteresis compensation mechanism uses an

Manuscript received November 2, 2008; revised March 24, 2009. First published June 19, 2009; current version published August 14, 2009. Recommended by Guest Editor P. X. Liu. The work of J. Yi was supported in part by the San Diego State Research Foundation and in part by the National Science Foundation under Grant CMMI-0826532. This work was presented at the 2009 American Control Conference, St. Louis, MO, June 10-12, 2009.

J. Yi is with the Department of Mechanical and Aerospace Engineering, Rutgers University, Piscataway, NJ 08854 USA (e-mail: jgyi@ rutgers.edu).

S. Chang is with the Department of Mechanical Engineering, San Diego State University, San Diego, CA 92182 USA (e-mail: changs@rohan.sdsu.edu).

Y. Shen is with the Department of Electrical and Biomedical Engineering, University of Nevada, Reno, NV 89557 USA (e-mail: ytshen@unr.edu).

Color versions of one or more of the figures in this paper are available online at http://ieeexplore.ieee.org.

Digital Object Identifier 10.1109/TMECH.2009.2023986 inverse hysteresis model in the feedforward loop to cancel the hysteresis nonlinearity. While this implementation does not require displacement or force sensors, an accurate mathematical model for hysteresis is essential to such an approach. Additionally, the hysteresis model is typically complicated [7], [8], and the method is prone to robustness issues due to disturbances. On the other hand, although the feedback hysteresis compensation approach requires external displacement or force sensors, it provides an effective and robust means to suppress the hysteresis nonlinearity. Moreover, the feedback approach does not rely on a precise mathematical model of the hysteresis.

We present a novel hysteresis compensation mechanism for a piezoelectric cantilever actuator used for micromanipulation applications. Our approach is based on the closed-loop compensation mechanism. Instead of precisely modeling the hysteresis, we treat the hysteresis as an external disturbance added to the linear dynamic behavior of the cantilever actuator. Then, we use a disturbance observer (DOB) to estimate and compensate for the hysteresis nonlinearity. The significance of the proposed DOB-based hysteresis compensation is its simplicity in implementation and robustness due to its independence from any hysteresis models. We have also experimentally validated the proposed compensation method on a piezoelectric cantilever actuator. The focus of this paper is to demonstrate the effectiveness, robustness, and simple implementation of the DOB-based compensation scheme. We leave the comparison of the proposed compensation with other existing model-based feedback/feedforward methods to ongoing future work.

Our approach is inspired by several related work [9], [11]. A DOB-based nonlinearity cancellation is proposed in [9] for a large class of single-input single-output (SISO) nonlinear systems. The output of the nonlinear SISO system is assumed to be the sum of the outputs of a stable SISO linear-time-invariant system and a bounded function of time. The nonlinearity in the system, for example, could include dead zone, backlash, and hysteresis. Our work is an extension of the approach in [9] and [10]. We use a piezoelectric cantilever actuator as an example to show that hysteresis nonlinearity can be decomposed into a bounded hysteresis operator and an approximated linear dynamic system. We also provide experimental validation.

A linear active disturbance rejection control (LADRC) is proposed in [11] for hysteresis compensation. Hysteresis nonlinearity is considered as a disturbance and a linear observer is proposed as a disturbance estimator. However, it is not clear why the hysteresis-induced disturbance can be treated as a linear system. Moreover, no experimental validation has been presented for the proposed approach. We relax the linear disturbance estimation 


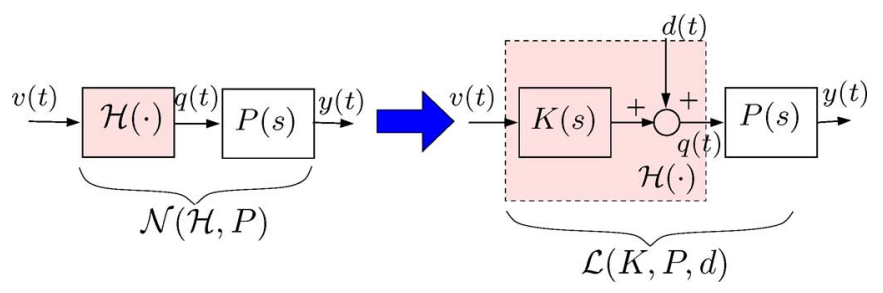

Fig. 1. Schematic of decomposition of a nonlinear system with hysteresis nonlinearity $\mathcal{H}(\cdot)$ as an integrated part of the system.

assumption and provide a more comprehensive treatment for the hysteresis nonlinearity.

The remainder of the paper is organized as follows. We present the DOB-based hysteresis compensation design in Section II. In Section III, we discuss the fabrication and modeling of a piezoelectric cantilever actuator. The experimental implementation of the DOB-based hysteresis compensator for the piezoelectric cantilever actuator is presented in Section IV. Finally, we conclude the paper in Section V.

\section{DOB-BASED HYSTERESIS COMPENSATION}

In this section, we first discuss the decomposition of a class of nonlinear dynamic systems with hysteresis relationship as a component of the system. Then, we present a DOB-based hysteresis compensation mechanism.

\section{A. Decomposition of a Class of Nonlinear Dynamic Systems With Hysteresis}

We consider here a class of nonlinear dynamic systems in which the hysteresis nonlinear relationship is one part of the dynamics. For the piezoelectric cantilever actuator that we will discuss in the next section, the applied voltage input $v(t)$ produces a charge $q(t)$ between the electrodes. This charge then produces a deformation in the piezoelectric material and the accompanying tip deflection $y(t)$. The left subfigure in Fig. 1 shows such a nonlinear relationship. It is well-known that a hysteresis relationship exists between the input voltage $v(t)$ and the charge $q(t)$ for piezoelectric materials [5]. We denote the hysteresis relationship between $v(t)$ and $q(t)$ as

$$
q(t)=(\mathcal{H}(v))(t) .
$$

The dynamic relationship between $q(t)$ and displacement $y(t)$ at the tip of the cantilever is given by the linear time-invariant system $P(s)$. We will discuss the development of $P(s)$ for the cantilever piezoelectric actuator example in the next section.

Let $\mathcal{N}(\mathcal{H}, P)$ denote the nonlinear dynamic system between input $v(t)$ and output $y(t)$. We propose to decompose $\mathcal{N}(\mathcal{H}, P)$ into a linear system $\mathcal{L}(K, P, d)$, as shown in the right subfigure in Fig. 1. Here, $K(s)$ denotes a linear time-invariant relationship between input variable $v(t)$ and intermediate variable $q(t)$. Also, the disturbance $d(t)$ is a bounded nonlinear function of input $v(t)$. We assume that the intermediate variable $q(t)$ is not measurable.

To further elucidate the earlier decomposition, we consider a Duhem model to capture the rate-independent hysteresis relationship between the input $v(t)$ and intermediate variable

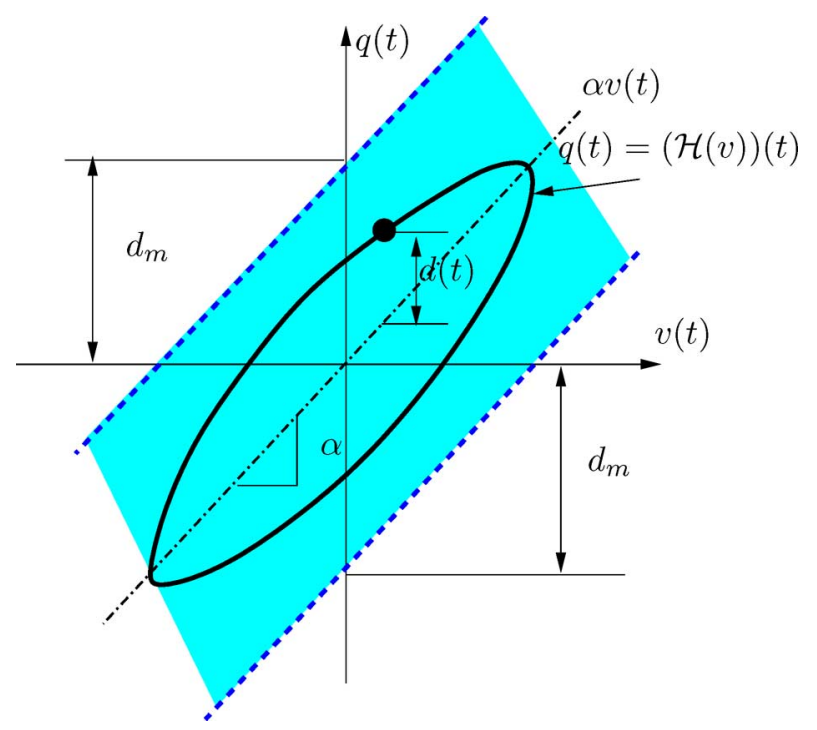

Fig. 2. Schematic of the linear representation for the hysteresis operator $q(t)=(\mathcal{H}(v))(t)=\alpha v(t)+d(t)$.

$q(t)$ [8], [12]. The Duhem model represents $\mathcal{H}(\cdot)$ by a firstorder nonlinear differential equation as

$$
\dot{q}(t)=\alpha|\dot{v}(t)|(a v(t)-q(t))+b \dot{v}(t)
$$

where $\alpha>0$ and $a>b \geq 1 / 2 a$ are model constants that depend on the shape and area of the hysteresis curves. In (2), $q(t)$ is considered as the state variable of the differential equation and depends on the values of both $v(t)$ and $\dot{v}(t)$. Such a mathematical relationship (2) can reproduce the hysteresis phenomena that we observe in experiments. Readers can refer to [8] and [12] for more details on how to estimate these hysteresis model parameters using experimental data.

We consider the solution properties of the Duhem dynamic model (2). Following a similar derivation in [13], we solve (2) explicitly as follows:

$$
q(t)=\alpha v(t)+d(t)
$$

where

$$
\begin{aligned}
d(t):=d(v(t))= & \left(q_{0}-a v_{0}\right) e^{-\alpha\left(v-v_{0}\right) \operatorname{sgn}(\dot{v})} \\
& +e^{-\alpha v \operatorname{sgn}(\dot{v})} \int_{v_{0}}^{v}(b-a) e^{\alpha \tau \operatorname{sgn}(\dot{v})} d \tau
\end{aligned}
$$

$q_{0}:=q(0), v_{0}:=v(0)$, and function $\operatorname{sgn}(x)=1$ if $x \geq 0$ and $\operatorname{sgn}(x)=-1$ if $x<0$. It is straightforward to check that for $\dot{v}>0$ or $\dot{v}<0$, the previous solution satisfies

$$
\lim _{v \rightarrow+\infty} d(t)=-\frac{a-b}{\alpha} \quad \text { or } \quad \lim _{v \rightarrow-\infty} d(t)=\frac{a-b}{\alpha}
$$

respectively. Therefore, $d(t)$ is bounded, that is, $|d(t)| \leq d_{m}:=$ $\sup _{t \geq 0}|d(t)|$.

Fig. 2 shows the linear representation of the nonlinear hysteresis operator $\mathcal{H}(\cdot)$ by (3) with the bounded unknown disturbance $d(t)$. Note that for the Duhem model

$$
K(s)=\alpha
$$

for the linear system $\mathcal{L}(K, P, d)$ shown in Fig. 1 . 


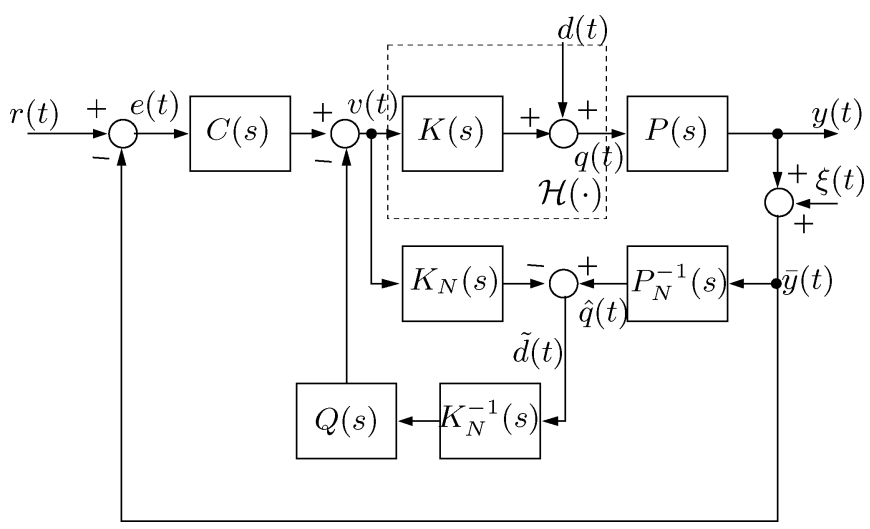

Fig. 3. Schematic of the DOB-based control design.

Although the decomposition analysis is proved using the Duhem hysteresis model, it is possible to show a similar treatment for other hysteresis models. With the earlier decomposition, we are now ready to apply the DOB scheme for the nonlinear hysteretic dynamic system (1).

\section{B. DOB-Based Hysteresis Compensation}

The general idea of DOB-based compensation is to estimate any unknown disturbances introduced into a system [14], [15]. The design of the DOB is accomplished through augmenting autonomous dynamical systems to the controlled plant. Because the DOB-based compensation is mainly utilized for disturbance rejection, additional feedback or feedforward controllers may be included to achieve the desired tracking performance if needed [16].

Fig. 3 shows the block diagram of the DOB-based hysteresis compensation mechanism [9]. Here, $C(s)$ denotes the controller for the plant $\mathcal{L}(K, P, d)$ and $\xi(t)$ denotes the measurement noise. $K_{N}(s)$ and $P_{N}(s)$ denote the nominal transfer functions for $K(s)$ and $P(s)$, respectively. It is straightforward to calculate that the estimated disturbance $\tilde{d}(t)$ will be close to $d(t)$ if the measurement noise $\xi(t)$ is negligible, and the nominal plants $K_{N}(s)$ and $P_{N}(s)$ are close to $K(s)$ and $P(s)$, respectively.

The filter $Q(s)$ is used to make $Q(s) K_{N}^{-1}(s) P_{N}^{-1}(s)$ realizable since $K_{N}^{-1}(s)$ and $P_{N}^{-1}(s)$ are typically noncasual. The relative degree of $Q(s)$ must be greater than or equal to that of the nominal plant $K_{N}(s) P_{N}(s)$ [15]. Moreover, all unstable zeros of $K_{N}(s) P_{N}(s)$ must be zeros of $Q(s)$ [17]. Typically, $Q(s)$ can be chosen as [15]

$$
Q(s):=Q(s ; \tau)=\frac{N_{Q}(s ; \tau)}{D_{Q}(s ; \tau)}=\frac{\sum_{k=1}^{N-r_{d}} \beta_{k}(\tau s)^{k}+1}{\sum_{k=1}^{N} \beta_{k}(\tau s)^{k}+1}
$$

where $r_{d}$ is the relative degree of $Q(s)$ and $\tau$ is the time constant of the filter.

The stability of the DOB-based control design has been recently presented in [16] and [18]. It has been shown in [16] that for certain disturbances, the DOB design is equivalent to the internal model principle. Particularly, for robot control, the DOBbased control is the same as the passivity-based approaches discussed in the aforementioned papers. Also, because of these

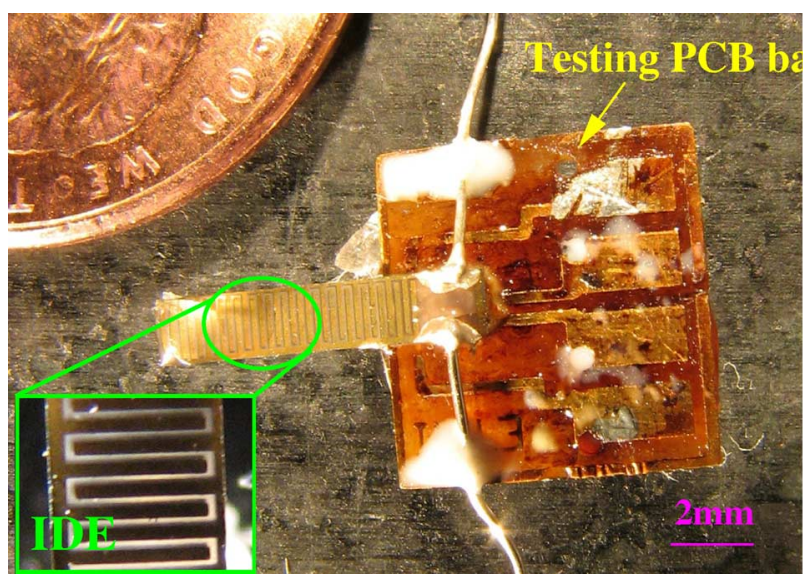

Fig. 4. PMN-PT/PDMS cantilever actuator.

equivalences, the stability and robustness of the DOB design can be obtained using the same methods. In the following, we briefly present a recent result in robust stability [18] that will be used in our hysteresis compensation design.

Consider the plant $P(s) \in \mathscr{P}$, where the set $\mathscr{P}$ is defined as a collection of transfer functions for which the leading coefficients of the numerator and denominator do not change sign. We define the robust internal stability for each $P(s) \in \mathscr{P}$, as the closedloop systems from the input set $[r(t) d(t) \xi(t)]^{T}$ to the output set $[e(t) v(t) \bar{y}(t)]^{T}$ (Fig. 3) that are stable under the DOBbased control systems design. We have the following stability results.

Proposition 1 ([18]): For $P(s) \in \mathscr{P}$, there exists a constant $\tau^{*}>0$ such that, for all $0<\tau<\tau^{*}$, the closed-loop system under the DOB-based control shown in Fig. 3 is robust internally stable if the following conditions hold: 1) $P_{N}(s) C(s) /(1+$ $\left.P_{N}(s) C(s)\right)$ is stable, 2) $P(s)$ is minimum phase, and 3) $P_{f}(s)$ is Hurwitz, where

$$
P_{f}(s):=D_{Q}(s ; 1)+\left(\lim _{s \rightarrow \infty} \frac{P(s)}{P_{N}(s)}-1\right) N_{Q}(s ; 1) .
$$

Note that it is possible to design the filter $Q(s)$ to meet the third condition in Proposition 1 [18].

\section{ACtuAtor Modeling AND StABility OF THE DOB-BASED CONTROL DESIGN}

In this section, we first describe the fabrication of the piezoelectric cantilever actuator that is used as the testing device for the proposed hysteresis compensation. We then present a dynamic model of the actuator. Finally, we briefly check the robust stability conditions presented in Proposition 1.

\section{A. Actuator Fabrication}

Fig. 4 shows a prototype of the piezoelectric cantilever actuator. We use lead magnesium niobate-lead titanate (PMNPT), a single crystal relaxor ferroelectric material, as the actuator material. The PMN-PT piezoelectric actuator was originally developed for microscale tip-based nanopowder sintering processes at San Diego State University. We also use an 
interdigitated electrode (IDE) design for better actuation performance. To reduce the stress concentration at the support, and therefore, increase the reliability of the actuator for cyclic loading, we use polydimethylsiloxane (PDMS) to form a coating layer on the PMN-PT cantilever. A proof mass at the cantilever tip is also formed by PDMS to emulate any attached endeffector, such as a conductive tip, for micro/nanomanipulation. A similar cantilever PMN-PT/PDMS design is also used as an energy harvester [19].

To fabricate the actuator prototype, we use $\langle 001\rangle$-factoryoriented PMN-PT single crystal plate with a thickness of $110 \mu \mathrm{m}$. The PMN-PT plate is polished and a thin layer (50 $\mathrm{nm}$ ) of gold or $\mathrm{Cr}$ is deposited on the top and the bottom surfaces to form fine primary electrode layers. The IDE patterns are custom fabricated by photolithography. The PMN-PT cantilever is then attached to a PCB board $(8 \mathrm{~mm} \times 8 \mathrm{~mm} \times 1$ $\mathrm{mm}$ ), as shown in Fig. 4. A PDMS epoxy coat is applied to the cantilever. Any air cavities are carefully removed. The PMNPT/PDMS cantilever is then heated to cure the PDMS epoxy. Once the PDMS has cured and hardened, it is cooled down to room temperature. A similar process is used to add the proof mass. Finally, the PMN-PT/PDMS cantilever undergoes poling.

\section{B. Dynamic Modeling}

For the purpose of modeling, the actuator prototype is considered as a composite cantilever with a point mass on its tip (see Fig. 5). Let $l$ and $b$ denote length and width of the PMNPT/PDMS composite cantilever, respectively. The mass of the proof mass is denoted by $M_{p}$. The heights of the PMN-PT and PDMS layers are denoted by $h_{p}$ and $h_{b}$, respectively. The mass density per unit length for the PMN-PT and the base beams are denoted by $\rho_{p}$ and $\rho_{b}$, respectively.

We consider the composite layer of the PMN-PT and PDMS as a single beam with flexural rigidity $E I_{\text {eq }}$ [19]. The mass density per unit length for the composite beam is $m:=\left(\rho_{p} h_{p}+\rho_{b} h_{b}\right) /\left(h_{p}+h_{b}\right)$. The vertical deflection of the beam is denoted by $w(x, t)$ at location $x$ and time $t$ in the coordinate system $x o z$ that is located on the base (see Fig. 5). The kinetic and potential energy of the system are

$$
\begin{aligned}
T & =\frac{1}{2} \int_{0}^{l} m \dot{w}(x, t)^{2} d x+\frac{1}{2} M_{p} \dot{w}(l, t)^{2} \\
V & =\frac{1}{2} \int_{0}^{l} E I_{\mathrm{eq}}\left(w^{\prime \prime}\right)^{2} d x
\end{aligned}
$$

where $\dot{w}(x, t):=\partial w(x, t) / \partial t$ and $w^{\prime \prime}(x, t):=\partial^{2} w(x, t) / \partial x^{2}$. Let $c$ denote the viscous damping coefficient for the beam. The virtual work done by the damping force is

$$
\delta W_{c}=\int_{0}^{l}\left(-c \dot{w}(x, t)+M_{n}^{\prime \prime}(x, t)\right) \delta w d x
$$

where $\delta w$ is the virtual displacement. The bending moment $M_{n}(x, t)$ due to the electric field by the input charge $q_{B}(t)$ (bottom electrode) and $q_{T}(t)$ (top electrode) is approximated

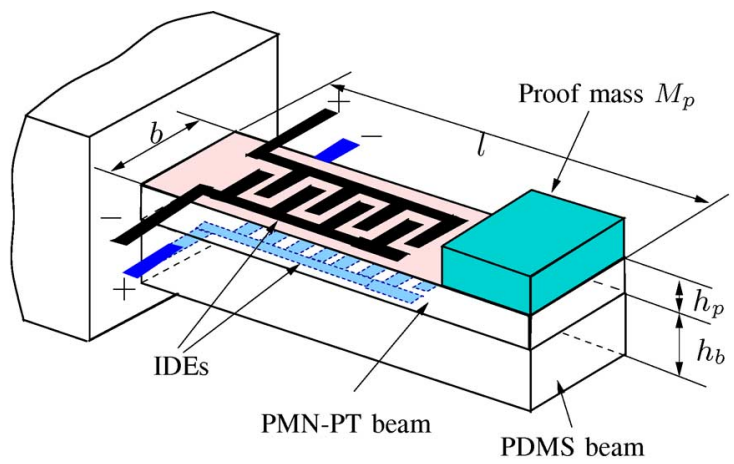

(a)
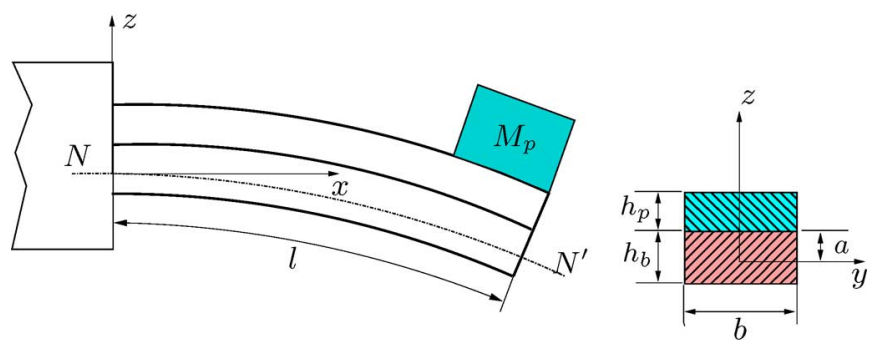

(b)

Fig. 5. (a) Operating diagram of the PMN-PT/PDMS actuator with IDEs. (b) 1-D schematic of the composite cantilever.

$[20]$ as

$$
M_{n}(x, t)=-\frac{E b d_{33} C_{E} h^{2}}{12 h_{F}} q_{d}(t)=K_{e} q_{d}(t)
$$

where $q_{d}(t):=q_{T}(t)-q_{B}(t), \quad K_{e}:=-E b d_{33} C_{E} h^{2} / 12 h_{F}$, $C_{E}$ is the capacitance of the IDE, $h_{F}$ is the interelectrode distance, and $d_{33}$ is the piezoelectric coefficient. We assume that the electrode starts at location $x_{s} \geq 0$ and ends at location $x_{e} \leq l$. Therefore, the spatial derivative $M_{n}^{\prime \prime}(x, t)$ can be represented as

$$
\begin{aligned}
M_{n}^{\prime \prime}(x, t) & =K_{e}\left[\delta^{\prime}\left(x-x_{s}\right)-\delta^{\prime}\left(x-x_{e}\right)\right] q_{d}(t) \\
& =K_{a} q_{d}(t)
\end{aligned}
$$

where $K_{a}=K_{e}\left[\delta^{\prime}\left(x-x_{s}\right)-\delta^{\prime}\left(x-x_{e}\right)\right]$, and $\delta^{\prime}(\cdot)$, the spatial derivative of the Dirac delta function, represents the unit dipole function [21].

Using the extended Hamilton's principles, we obtain the equations of motion and boundary conditions

$$
\begin{aligned}
& M_{p} \frac{\partial^{2} w(l, t)}{\partial t^{2}}+m \frac{\partial^{2} w}{\partial t^{2}}+c \frac{\partial w}{\partial t}+E I_{\mathrm{eq}} \frac{\partial^{4} w}{\partial x^{4}}=M_{n}^{\prime \prime}(x, t) \\
& M_{p} \frac{\partial^{2} w(l, t)}{\partial t^{2}}-E I_{\mathrm{eq}} \frac{\partial^{3} w(l, t)}{\partial x^{3}}=0
\end{aligned}
$$

$w(0, t)=w^{\prime}(0, t)=w^{\prime \prime}(l, t)=0$.

We define $\xi:=x / l, \beta:=M_{p} / m l$, and $\lambda^{4}:=m l^{4} \omega^{2} / E I_{\mathrm{eq}}$ as the dimensionless length of the beam, mass ratio of the proof mass and the composite beam, and the natural frequency parameters of the beam-mass system, respectively. The characteristic equation of the dynamic systems given in (8) is then obtained as

$$
1+\cos \lambda \cosh \lambda+\lambda \beta(\cos \lambda \sinh \lambda-\sin \lambda \cosh \lambda)=0 .
$$


The natural frequency decreases as $\beta$ (or as the tip mass) increases [19].

By variable separation, we write $w(x, t)=\sum_{i=1}^{\infty} \phi_{i}(x) p_{i}(t)$, where $\phi_{i}(x)$ is the $i$ th modal shape and $p_{i}(t)$ is the $i$ th generalized coordinate for the system. For $i$ th mode, the equation of motion for the beam-mass system in terms of $p_{i}(t)$ is obtained as

$$
m_{e i} \ddot{p}_{i}(t)+c_{e i} \dot{p}_{i}(t)+k_{e i} p_{i}(t)=f_{e i} q_{d}(t)
$$

where the generalized mass, damping, and stiffness coefficients are

$$
\begin{aligned}
m_{e i} & =\int_{0}^{l} m \phi_{i}^{2}(x) d x+M_{p} \phi_{i}^{2}(l) \\
c_{e i} & =\int_{0}^{l} c \phi_{i}^{2}(x) d x \\
k_{e i} & =\int_{0}^{l} E I_{\mathrm{eq}}\left(\phi_{i}^{\prime \prime}(x)\right)^{2} d x
\end{aligned}
$$

respectively. The generalized force coefficient is given by

$$
f_{e i}=\int_{0}^{l} K_{a} \phi_{i}(x) d x=K_{e}\left[\frac{d \phi_{i}}{d x}\left(x_{s}\right)-\frac{d \phi_{i}}{d x}\left(x_{e}\right)\right] .
$$

Let $w_{p}(t):=w(l, t)=\sum_{i=1}^{\infty} \phi_{i}(l) p_{i}(t)$ denote the measurable tip displacement. Taking Laplace transformation, from (10), we find the transfer function between the excitation charge $q_{d}(t)$ and the tip displacement $w_{p}(t)$ as

$$
P(s):=\frac{W_{p}(s)}{Q_{d}(s)}=\sum_{i=1}^{\infty} P_{i}(s)=\sum_{i=1}^{\infty} \frac{A_{v i} \omega_{n i}^{2}}{s^{2}+2 \zeta_{i} \omega_{n i} s+\omega_{n i}^{2}}
$$

where $\omega_{n i}^{2}=k_{e i} / m_{e i}, \quad 2 \zeta_{i} \omega_{n i}=c_{e i} / m_{e i}, \quad$ and $\quad A_{v i}=$ $\phi_{i}(l) f_{e i} / k_{e i}$.

Remark 1: In the previous analysis, we neglect the nonlinear effects of the deformation of the composite beam and the electromechanical characteristic of the PMN-PT piezoelectric material. The treatment of these nonlinear properties on dynamical modeling can be found in [22]. The damping effect due to the electromechanical coupling of the piezoelectric material is also neglected in the models.

\section{Robust Stability of the DOB-Based Control Design}

Since the transfer function (11) shows the system to exhibit vibrational behavior, it is appropriate to use the lowest mode to approximate the dynamics for applications utilizing a low frequency range. Therefore, we take the first mode as the approximation for the plant dynamics in the DOB-based hysteresis control systems design, namely,

$$
P_{N}(s)=P_{1}(s)=\frac{A_{v 1} \omega_{n 1}^{2}}{s^{2}+2 \zeta_{1} \omega_{n 1} s+\omega_{n 1}^{2}} .
$$

Since the plant dynamics $P(s)$ in (11) is stable and minimumphase with relative degree of 2 , we choose a second-order Butterworth filter for $Q(s)$

$$
Q(s)=Q(s ; \tau)=\frac{1}{(\tau s)^{2}+\sqrt{2} \tau s+1}
$$

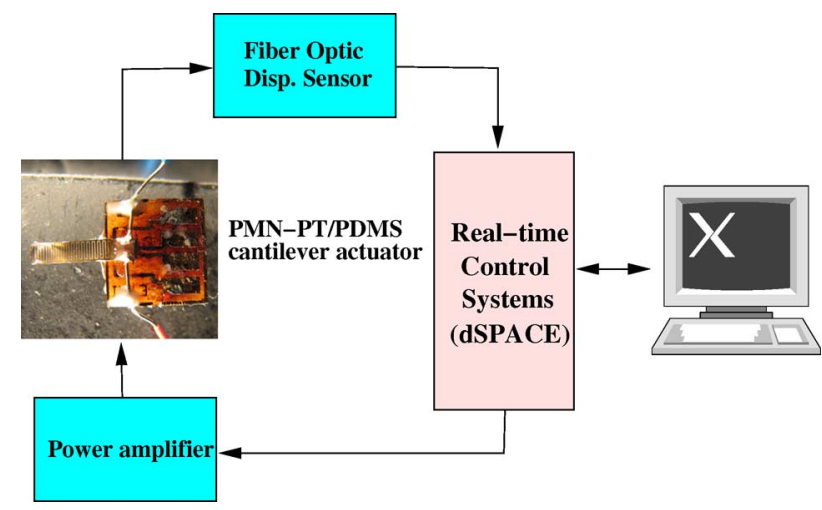

Fig. 6. Schematic of the testing systems.

TABLE I

Estimated AND MEASURED First AND SECOND NatURAL FrEQUENCIES AND THE DAMPING COEFFICIENT $\zeta_{1}$ OF THE ACTUATOR

\begin{tabular}{|c|c|c|c|}
\hline \hline & Analytical $\frac{\omega_{n i}}{2 \pi}(\mathrm{Hz})$ & Exp. $\frac{\omega_{n i}}{2 \pi}(\mathrm{Hz})$ & Estimated coeff. $\zeta_{i}$ \\
\hline 1st mode & 1552 & 1547 & 0.00876 \\
\hline 2nd mode & 10977 & 9550 & - \\
\hline \hline
\end{tabular}

where $\tau=1 / \omega_{c}$ and $\omega_{c}$ is the cutoff frequency of the filter $Q(s)$. Note that the relative degree of $Q(s)$ is the same as that of the plant $P(s) . Q(s)$ is chosen as a low-pass filter that admits the lower frequencies used in disturbance rejection while rejecting higher frequencies associated with sensor noises [16].

To check the stability conditions given in Proposition 1, the first condition can easily be satisfied by designing the feedback controller $C(s)$ such that the closed-loop systems with the nominal plant $P_{N}(s)$ is stable. For the second condition, $P(s)$ is minimum phase and stable given the fact that the gain of the first mode is relatively large and the cantilever piezoelectric beam is a stable system. To check the third condition, we note that

$$
\lim _{s \rightarrow \infty} \frac{P(s)}{P_{N}(s)}-1=\lim _{s \rightarrow \infty} \sum_{i=2}^{\infty} \frac{P_{i}(s)}{P_{1}(s)}=\sum_{i=2}^{\infty} \frac{A_{v i} \omega_{n i}^{2}}{A_{v 1} \omega_{n 1}^{2}}=: R_{p}
$$

and therefore

$$
P_{f}(s)=D_{Q}(s ; 1)=s^{2}+\sqrt{2} s+\left(1-R_{p}\right) .
$$

Since typically $A_{v i} \ll A_{v 1}$ from vibration theory, we assume without loss of generality ${ }^{1}$ that $R_{p}<1$. Therefore, $P_{f}(s)$ is Hurwitz and the robust internal stability can be obtained by Proposition 1.

\section{EXPERIMENTS}

\section{A. Experimental Setup}

A PMN-PT cantilever actuator prototype (shown in Fig. 4) is fabricated with a dimension of $7.4 \mathrm{~mm} \times 2 \mathrm{~mm} \times 110 \mu \mathrm{m}$. To test the cantilever actuator, we set up a testbed, as shown in Fig. 6. The cantilever actuator is mounted on a fixed base. A high-precision fiber optic displacement sensor system (model

\footnotetext{
${ }^{1}$ If $R_{p} \geq 1$, we can always redesign the constant term of $D_{Q}(s ; 1)$ to make it larger than $R_{p}$. A similar explanation is also presented in [18].
} 


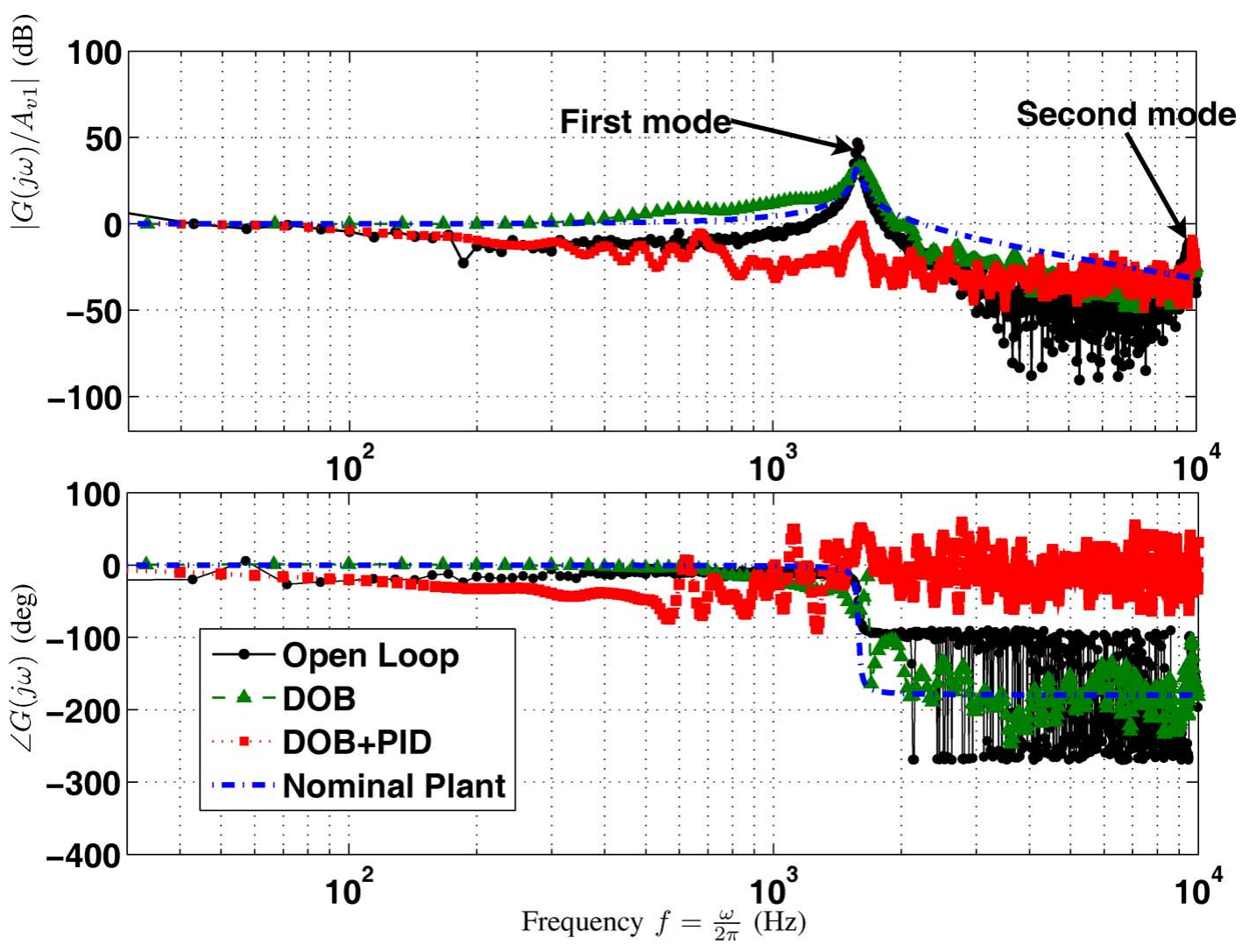

Fig. 7. Bode plots of the cantilever actuator.

D11, Philtec, Inc.) is used to measure the tip displacement of the cantilever. The output sensitivity of the fiber optic displacement sensor is $12.3 \mathrm{~nm} / \mathrm{mV}$. The cantilever actuator is driven by an amplifier (model 790A, PCB Piezotronics, Inc.). A realtime control system (model ACE1104, dSPACE, Inc.) is used to control the motion of the actuator and also to collect the displacement data.

\section{B. Estimation of Model Parameters}

The estimation of the actuator parameters, such as geometry and mechanical properties, can be found in [19]. With these parameters, we can analytically estimate the first and second natural frequencies of the actuator using (9), as shown in Table I. The Bode plot of the transfer function $P(s)$ is shown in Fig. 7. We obtain the frequency response by impulse excitation input. The estimated first and second natural frequencies are also listed in Table I. The estimates of these natural frequencies from the Bode plots match the analysis closely. Note that the Bode plot of the nominal plant $P_{N}(s)$ shown in Fig. 7 matches the frequency responses of $P(s)$ at low frequencies. We also obtained an estimate of the damping coefficient $\zeta_{1}$ through the step response of the system [19]. The estimated value is listed in Table I.

For our experiments, we only excite the electrodes on the top surface of the actuator. The bottom electrodes are connected to ground. Because we cannot measure the charge signal $q(t)$ in experiments, we cannot directly determine the parameter $A_{v}$ in the plant model (11). However, from an input/output perspec- tive, we can treat $A_{v}=1 \mathrm{~nm} / \mathrm{C}$ and let the parameter $\alpha$ in the hysteresis operator (3) determine the dc gain of the system.

Fig. 8(a) and (c) show the actuator response under a sinusoidal input with a frequency of 10 and $100 \mathrm{~Hz}$, respectively. Clearly, the hysteresis nonlinearity in actuator response is independent of input frequency. We estimate the value of $\alpha$ by taking an average of the estimates from various input frequencies, and the following estimate is obtained:

$$
K(s)=K_{N}(s)=\alpha=76.4 \mathrm{C} / \mathrm{V} .
$$

\section{Hysteresis Compensation Performance}

To demonstrate the effectiveness of the DOB-based hysteresis compensation, we show several experimental examples under various input excitations. Fig. 8(b) and (d) show the relationship of the tip displacement versus the input voltage under DOB compensation without the use of additional feedback controllers $C(s)$. In these experiments, we design the cutoff frequency $\omega_{c}$ of the DOB filter $Q(s)$ to be $500 \mathrm{~Hz}$ since we only conduct low frequency excitations. Although the effectiveness of the proposed compensation is only shown under two input frequencies, our testing results have demonstrated effectiveness of the compensation up to $150 \mathrm{~Hz}$. For these particular experiments, the DOB-based compensation was effective in removing all hysteresis detectable given the limitations of the sensors used. Fig. 9 shows a comparison of the tracking performance of the actuator under sinusoidal input with various frequencies. The desired tracking trajectory is a sinusoidal signal with an 


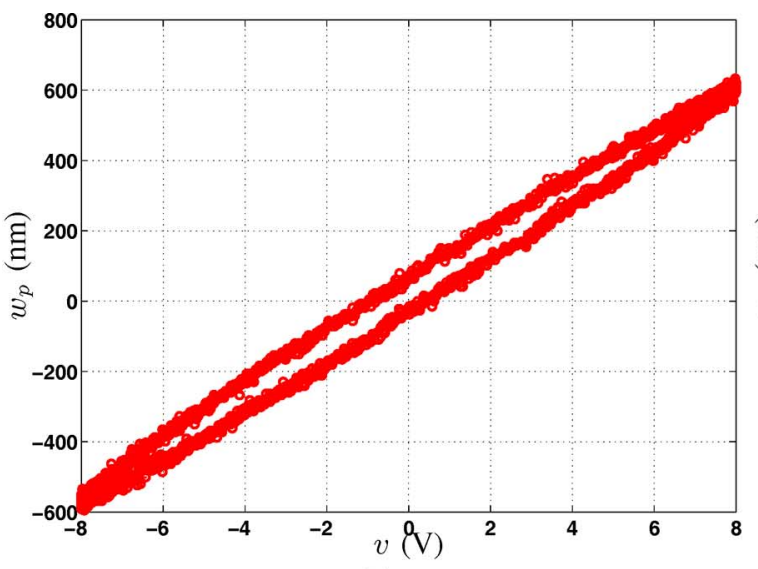

(a)

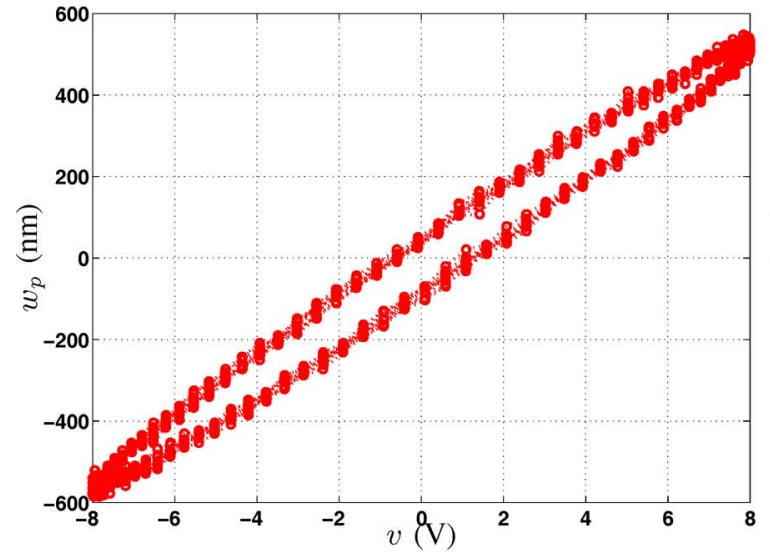

(c)

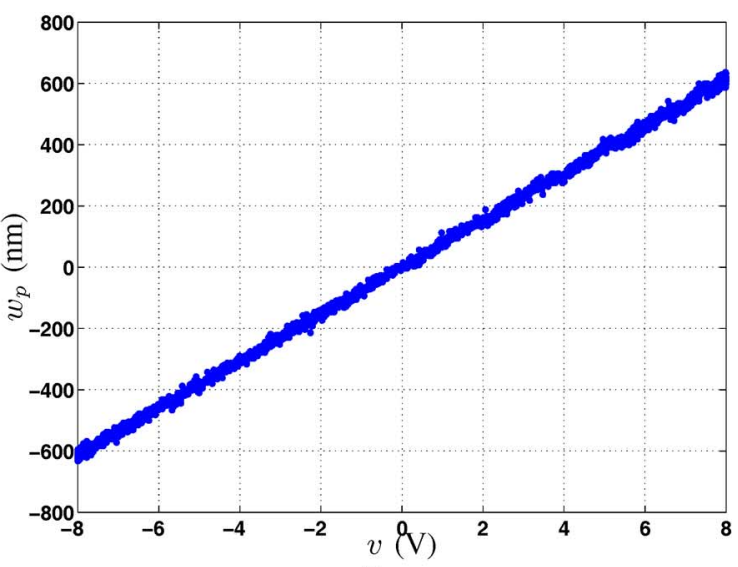

(b)

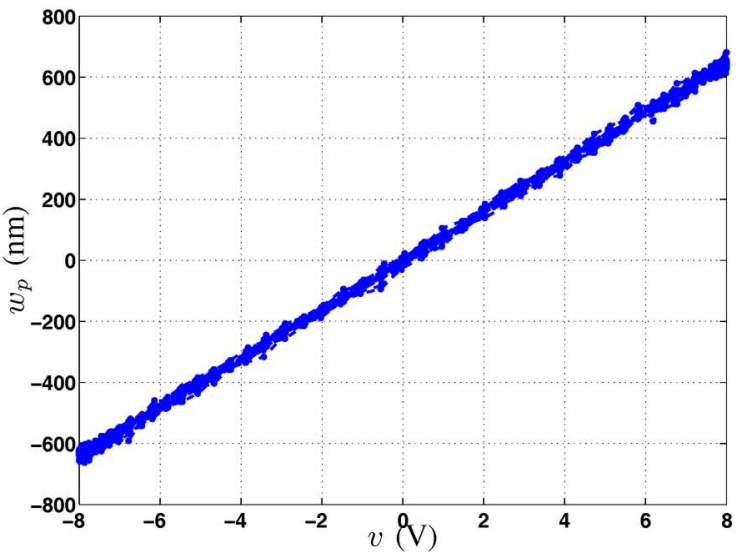

(d)

Fig. 8. Tip displacement versus the input voltage. (a) Without DOB under a 10-Hz excitation. (b) With DOB compensation under a 10-Hz excitation. (c) Without DOB under a $100-\mathrm{Hz}$ excitation. (d) With DOB compensation under a $100-\mathrm{Hz}$ excitation.

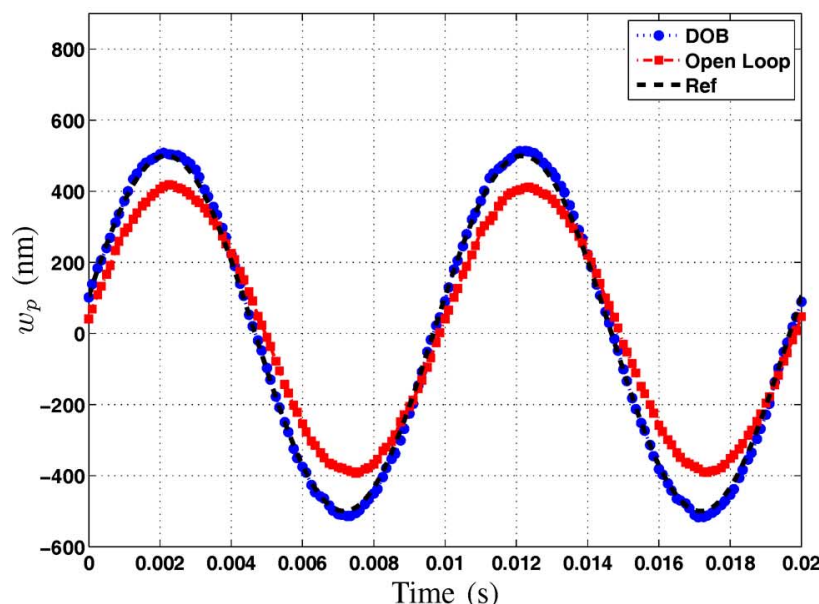

(a)

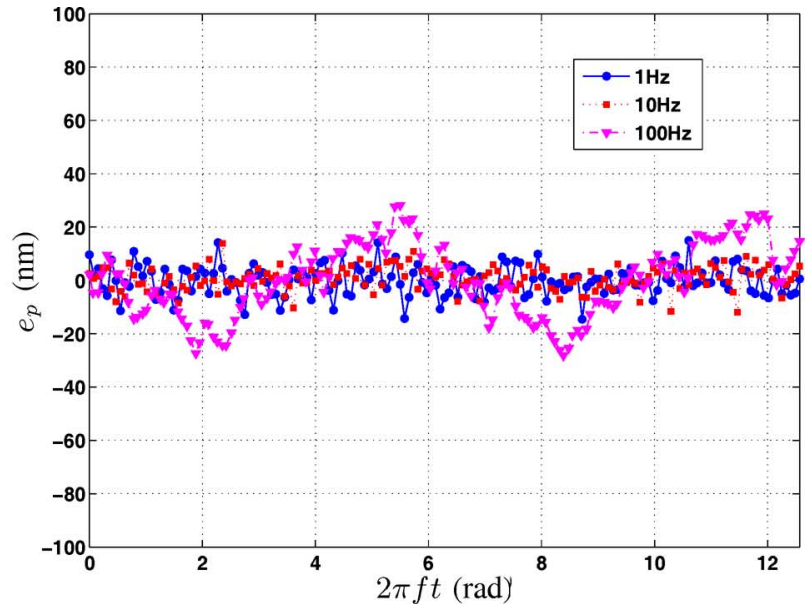

(b)

Fig. 9. (a) Comparison results of the tracking performance with and without DOB hysteresis compensation under a 100-Hz sinusoidal input. (b) Tracking errors $e_{p}(t)$ under sinusoidal inputs with various frequencies.

amplitude of $500 \mathrm{~nm}$ and frequencies of 1, 10, and $100 \mathrm{~Hz}$. For each case, we only compare the compensation performance with and without DOB. In Fig. 9(a), we illustrate the trajectory tracking performance with and without DOB compensation at 100-Hz input excitation, while Fig. 9(b) shows the tracking er- rors $e_{p}(t):=r(t)-y(t)$ under 1-, 10-, and 100-Hz frequencies inputs. We clearly see that without the DOB compensation, the tip displacement displays a "phase lag" with respect to the reference trajectory. With the DOB-based hysteresis compensation, the tip displacement follows the reference closely. It is also noted 


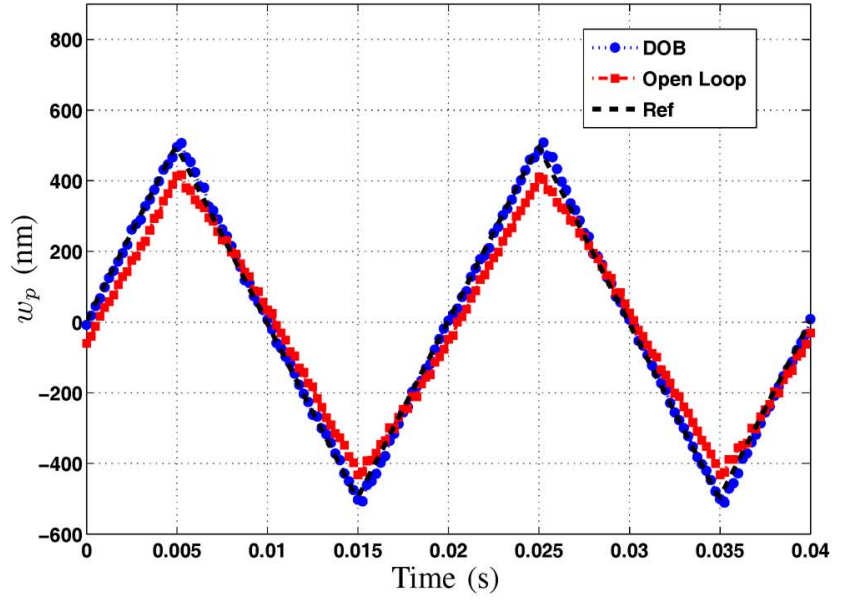

(a)

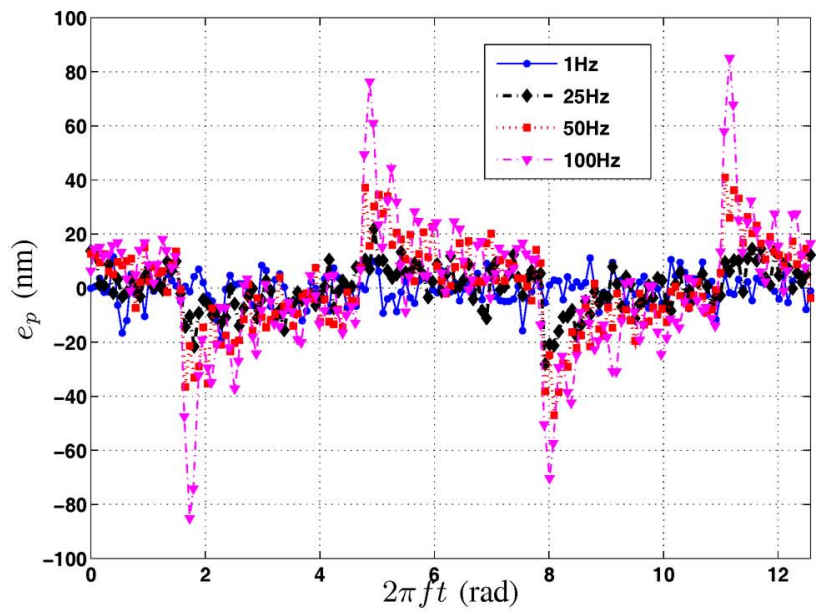

(b)

Fig. 10. (a) Comparison results of the tracking performance with and without DOB hysteresis compensation under a 50-Hz triangular-shape input. (b) Tracking errors $e_{p}(t)$ with various input frequencies.

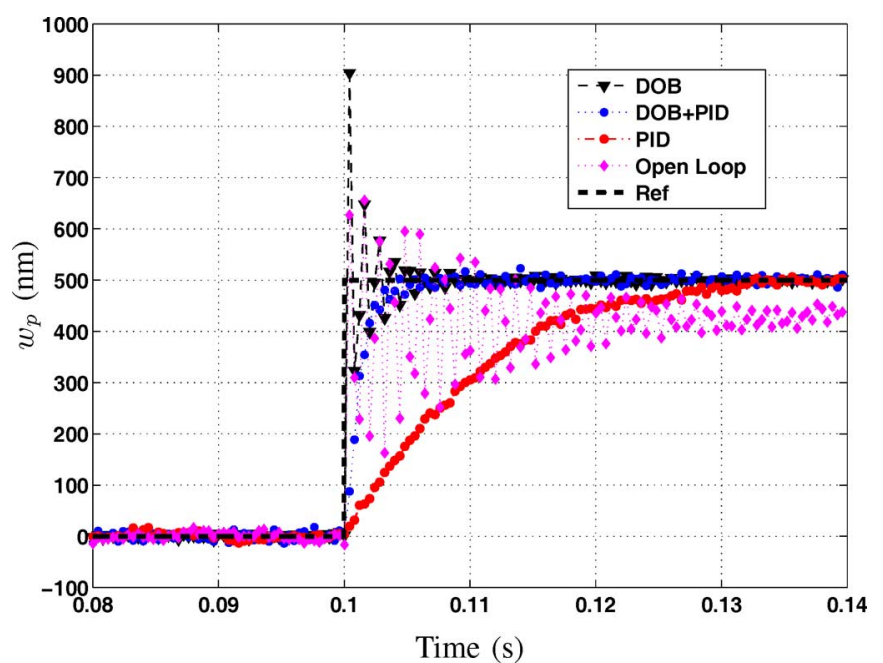

Fig. 11. Comparison results of the step responses with and without DOB hysteresis compensation.

that with increased input frequencies, the DOB-based tracking errors increase as well.

In Fig. 10, we demonstrate the tracking performance for periodic triangular input trajectories at various frequencies. Such input trajectories have been used for micropositioning systems such as tip motion under the scanning mode of AFM. Similar to the previous example, Fig. 10(a) shows the tracking performance under an input frequency of $50 \mathrm{~Hz}$, while Fig. 10(b) illustrates the tracking errors $e_{p}(t)$ at $1,25,50$, and $100 \mathrm{~Hz}$. These results clearly demonstrate that under only DOB compensation, the output displacements track the input trajectory closely.

As a last example, Fig. 11 shows the tracking performance of the system to a step input with and without the DOB-based hysteresis compensation. For these experiments, we introduce a PID controller $C(s)$ to track the reference signal $r(t)$. For the PID controller, we used the gains $K_{p}=10^{-4}, K_{i}=125$, and $K_{d}=10^{-10}$. These gains were selected to offer the best performance under a step input without DOB compensation. We also include the tracking performance of the system under open-loop as well as under only DOB compensation. We observe that in the open-loop case, the output exhibits oscillations. Furthermore, the output converges slowly with a steady state error of roughly $80 \mathrm{~nm}$. Under only PID control, the output does not exhibit oscillations; however, convergence is also slow due to the presence of hysteresis. In the case with only DOB compensation, the output converges quickly, but with considerable oscillations. Finally, with a combination of DOB and PID control, the output converges quickly and exhibits no oscillations.

\section{CONCLUSION}

In this paper, we demonstrated a novel hysteresis compensation method for piezoelectric actuators. The compensation mechanism treats the hysteresis nonlinearity as an unknown disturbance added to a linear system. As a result, the proposed hysteresis compensation method does not rely on any mathematical model of hysteresis. Simplicity in implementation and robustness of performance are the main advantages of the proposed DOB-based hysteresis compensation approach. We have demonstrated the effectiveness and efficiency of the method as applied to a PMN-PT cantilever piezoelectric actuator for micromanipulation applications. The experimental results confirmed that the proposed DOB-based compensation scheme successfully suppressed the hysteresis of the piezoelectric actuators to a satisfactory level under an input frequencies up to $150 \mathrm{~Hz}$.

In the future, we plan to further improve the DOB design and compare its performance with other feedback/feedforward hysteresis compensation methods. We are also interested in enhancing the DOB-based design for high-bandwidth hysteresis compensation that can be applied to micro/nanopositioning systems. 


\section{ACKNOWLEDGMENT}

The authors thank Dr. S. M. Shahruz at Berkeley Engineering Research Institute, Prof. K. S. Moon, Prof. K. Morsi, and Prof. S. Kassegne at San Diego State University for their helpful discussions. They are also grateful to A. Mathers for his help in fabricating the PMN-PT/PDMS cantilever.

\section{REFERENCES}

[1] T. Fukuda, F. Arai, and L. Dong, "Assembly of nanodevices with carbon nanotubes through nanorobotic manipulations," Proc. IEEE, vol. 91, no. 11 , pp. 1803-1818, Nov. 2003.

[2] N. Xi and W. Li, "Recent development in nanoscale manipulation and assembly," IEEE Trans. Autom. Sci. Eng., vol. 3, no. 3, pp. 194-198, Jul. 2006.

[3] S. Devasia, E. Eleftheriou, and S. Moheimani, "A survey of control issues in nanopositioning," IEEE Trans. Control Syst. Technol., vol. 15, no. 5, pp. 802-823, Sep. 2007.

[4] X. Tan and R. Iyer, "Modeling and control of hysteresis," IEEE Control Syst. Mag., vol. 29, no. 1, pp. 26-28, Feb. 2009.

[5] A. J. Fleming and S. O. R. Moheimani, "A grounded-load amplifier for reducing hysteresis in piezoelectric tube scanners," Rev. Sci. Instrum., vol. 76, pp. 073707-1-073707-5, 2005.

[6] H. Janocha, D. Pesotski, and K. Kuhnen, "FPGA-based compensator of hysteretic actuator nonlinearities for highly dynamic applications," IEEE/ASME Trans. Mechatronics, vol. 13, no. 1, pp. 112-116, Feb. 2008.

[7] J. Macki, P. Nistri, and P. Zecca, "Mathematical models for hysteresis," SIAM Rev., vol. 35, no. 1, pp. 94-123, 1993.

[8] A. Visintin, "Mathematical models of hysteresis," in The Science of Hysteresis, G. Bertotti and I. Mayergoyz, Eds. London, U.K.: Academic, 2006, pp. 1-123.

[9] S. Shahruz, "Performance enhancement of a class of nonlinear systems by disturbance observers," IEEE/ASME Trans. Mechatronics, vol. 5, no. 3, pp. 319-323, Sep. 2000.

[10] S. Chang, J. Yi, and Y. Shen, "Disturbance observer-based hysteresis compensation for piezoelectric micro-actuators," in Proc. Amer. Control Conf., St. Louis, MO, 2009, pp. 4196-4201.

[11] F. Goforth and Z. Gao, "An active disturbance rejection control solution for hysteresis compensation," in Proc. Amer. Control Conf., Seattle, WA, 2008, pp. 2202-2208.

[12] H. Adriaens, W. de Koning, and R. Banning, "Modeling piezoelectric actuators," IEEE/ASME Trans. Mechatronics, vol. 5, no. 4, pp. 331-341, Dec. 2000

[13] C.-Y. Su, Y. Stepanenko, J. Svoboda, and T. Leung, "Robust adpative control of a class of nonlinear systems with unknown backlash-like hysteresis," IEEE Trans. Autom. Control, vol. 45, no. 12, pp. 2427-2432, Dec. 2000.

[14] K. Ohishi, M. Nakao, K. Ohnishi, and K. Miyachi, "Microprocessorcontrolled DC motor for load-insensitive position servo system," IEEE Trans. Ind. Electron., vol. 34, no. 1, pp. 44-49, Feb. 1987.

[15] T. Umeno and Y. Hori, "Robust speed control of DC servomotors using modern two-degree-of-freedom controller design," IEEE Trans. Ind. Electron., vol. 38, no. 5, pp. 363-368, Oct. 1991.

[16] E. Schrijver and J. van Dijk, "Disturbance observers for rigid mechanical system: Equivalence, stability, and design," Trans. ASME, J. Dyn. Syst. Meas. Control, vol. 124, no. 1, pp. 539-548, 2002.

[17] A. Tesfaye, H. Lee, and M. Tomizuka, "A sensitivity optimization approach to design of a disturbance observer in digital motion control systems," IEEE/ASME Trans. Mechatronics, vol. 5, no. 1, pp. 32-38, Mar. 2000 .

[18] H. Shim and N. Jo, "An almost necessary and sufficient condition for robust stability of closed-loop systems with disturbance observer," Automatica, vol. 45, pp. 296-299, 2009.

[19] A. Mathers, K. S. Moon, and J. Yi, "A vibration-based PMN-PT energy harvester,” IEEE Sens. J., vol. 9, no. 7, pp. 731-739, Jul. 2009.

[20] J. Yi, S. Chang, K. Moon, and Y. Shi, "Dynamic modeling of an L-shape PMN-PT piezo-based manipulator," in Proc. Amer. Control Conf., Seattle, WA, 2008, pp. 3755-3760.

[21] H. R. Pota and T. E. Alberts, "Multivariable transfer functions for a slewing piezoelectric laminate beam," Trans. ASME, J. Dyn. Syst., Meas., Control, vol. 117 , no. 1 , pp. 352-359, 1995.
[22] S. Mahmoodi, N. Jalili, and M. Daqaq, "Modeling, nonlinear dynamics, and identification of a piezoelectrically actuated microcantilever sensor," IEEE/ASME Trans. Mechatronics, vol. 13, no. 1, pp. 58-65, Feb. 2008.

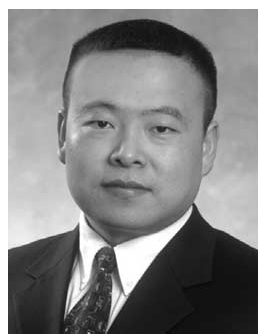

Jingang Yi (S'99-M'02-SM'07) received the B.S degree in electrical engineering from Zhejiang University, Hangzhou, China, in 1993, the M.Eng. degree in precision instruments from Tsinghua University, Beijing, China, in 1996, and the M.A. degree in mathematics and the Ph.D. degree in mechanical engineering from the University of California, Berkeley, in 2001 and 2002, respectively.

From May 2002 to January 2005, he was with Lam Research Corporation, Fremont, CA, as a Member of Technical Staff. From January 2005 to December 2006, he was with the Department of Mechanical Engineering, Texas A\&M University, as a Visiting Assistant Professor. Prior to joining Rutgers University in August 2008, he was an Assistant Professor of mechanical engineering at San Diego State University beginning in January 2007. He is currently an Assistant Professor of mechanical engineering at Rutgers University, Piscataway, NJ. His current research interests include autonomous robotic systems, dynamic systems and control, intelligent sensing and actuation systems, mechatronics, and automation science and engineering, with applications to semiconductor manufacturing, intelligent transportation, and biomedical systems.

Dr. Yi is a member of the American Society of Mechanical Engineers (ASME). He has coauthored papers that have been awarded the Best Student Paper Award Finalist of the 2008 ASME Dynamic Systems and Control Conference, the Best Conference Paper Award Finalists of the 2007 and 2008 IEEE International Conference on Automation Science and Engineering, and the Kayamori Best Paper Award of the 2005 IEEE International Conference on Robotics and Automation. He is currently an Associate Editor of the ASME Dynamic Systems and Control Division and the IEEE Robotics and Automation Society Conference Editorial Boards. He has also been a Guest Editor of the IEEE TRANSACTIONS ON AUTOMATION SCIENCE AND ENGINEERING.

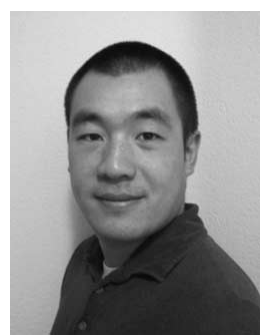

Steven Chang received the B.S. degree in mechanical engineering from the University of California, Berkeley, in 2003. He is currently working toward the M.S. degree in mechanical engineering with emphases in controls and mechatronics at San Diego State University, San Diego, CA.

He was a Technical Assistant with the Institute of Transportation Studies Pavement Research Center. From October 2004 to August 2007, he was a Product Quality Engineer before enrolling at San Diego State University.

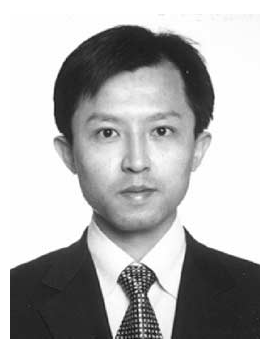

Yantao Shen (M'02) received the B.Eng. degree in mechanical and electronic engineering and the M.Eng. degree in mechatronic control and automation, both from Beijing Institute of Technology, Beijing, China, in 1994 and 1997, respectively, and the Ph.D. degree in sensor-based robotic systems from the Chinese University of Hong Kong, Hong Kong, in 2002.

From 2002 to 2007, he was a Research Associate in the Department of Electrical and Computer Engineering, Michigan State University. In January 2008, he joined the University of Nevada, Reno, where he is currently an Assistant Professor in the Department of Electrical and Biomedical Engineering. He has authored or coauthored more than 60 papers in professional journals and conference proceedings. His current research interests include the areas of bioinstrumentation, smart sensors and actuators for biosystems, sensorized micro/nanosystems, visual servo systems, and haptic/tactile interfaces.

Dr. Shen is a member of Sigma Xi. He is currently an Associate Editor for several conferences of the IEEE Robotics and Automation Society. He was also a finalist for the Best Vision Paper Award at the IEEE International Conference on Robotics and Automation in 2001. 